\title{
Approximate Entropy (ApEn) based Heart Rate Variability Analysis
}

\author{
Amritpal Singh ${ }^{1 *}$ and Jashandeep Kaur ${ }^{2}$ \\ ${ }^{1}$ School of Electronics and Electrical Engineering, Lovely Professional University, Phagwara - 144411, Punjab, India; \\ apsingh.lpu@gmail.com \\ ²Lovely Professional University, Phagwara - 144411, Punjab, India
}

\begin{abstract}
Heart Rate Variability (HRV) has been established as a vital index for diagnostics and prognosis of a number of pathological conditions. Moreover, $\mathrm{HRV}$ is a proven indicator of autonomic balance. As HRV is a result of multiple responses acting at various time scales, these interactions need to be quantified. In this paper, a complexity measure called ApEn is utilized to quantify the complexity of HRV. This method is tested on age stratified standard Fantasia database from Physionet. It is observed that young subjects show higher HRV complexity than the older ones. The effect of tolerance threshold ' $r$ ' is also evaluated on the HRV complexity estimation of young and old subjects. Further, for $r \geq 0.10$, the complexity of HRV is higher for young subjects but the trend is reverse for $r<0.10$. Therefore, it is concluded that the tolerance threshold ' $r$ ' should be carefully selected for the complexity analysis of HRV.
\end{abstract}

Keywords: Approximate Entropy, Autonomic Nervous System, Complexity, Heart Rate Variability

\section{Introduction}

Heart rate variability is one of the simple and widely accepted measures by cardiologist for both clinical and research use as reveals the cardiac health and Autonomic nervous system. Heart rate variability is defined as a physiological process of determining variability in time gap between consecutive heart beats. Heart rate variability is regulated by sinoatrial node having two parts sympathetic and parasympathetic nodes and is a natural pacemaker of human body. Beat-to-beat analysis of HRV provides an important information regarding cardiovascular tone non-invasively. The analysis of HRV is a noninvasive diagnostic tool that provides significant prognostic information in cardiology for both physiological and psychophysiological processes $\frac{1,2,14,15,16,17}{1}$. HRV is the result of modulation of both cardiac parasympathetic and sympathetic outflow to the sinus node in the heart. HRV is affected by a number of physiological and psychophysiological processes. These include body position or postural stress, mental stress, exercise, moderate hypo- tension, controlled respiration, and in various diseases like diabetes mellitus, myocardial infarction etc. . $^{3-13}$. In ${ }^{4}$ it has been mentioned that HRV is capable to discriminate amongst post myocardial infarction cardiac arrest (MI-CA) survivors from further post MI patients. HRV index is furthermore capable to measure autonomic dysfunction of diabetic patients ${ }^{5}$.

Over the last ten years, indices of HRV have arisen as a pronounced predictive pointer of extensive range of cardiovascular diseases ${ }^{6-9}$. Invasive and non-invasive approaches have been established over a period of time to examine HRV. The invasive approaches utilize outer stimulus, viz., drugs to stimulate or block one of the branches of Autonomic Nervous System (ANS) thereby altering the autonomic balance. This is in turn leads to changes in HRV. Non-invasive methods include signal processing techniques for time domain, frequency domain and Time-frequency analysis of HRV. For analysis of HRV, $\mathrm{RR}$ intervals (time between successive R-peaks) series obtained from electrocardiogram (ECG) signal is usually analyzed. Non-invasive ways of assessing HRV is

${ }^{*}$ Author for correspondence 
grounded on natural beat-to-beat measurement of heart rate. Statistical methods are used for analyzing HRV timedomain. Various parameters like SDNN, SDSD, RMSSD, SDNN index etc. are used for the time-domain analysis of HRV. Frequency domain approaches comprise parametric and non-parametric power spectral estimation to assess Very Low Frequency (VLF), Low Frequency (LF) and High Frequency (HF) power bands $\stackrel{7,8}{ }$. LF/HF ratio is an important index to assess sympathovagal balance. Since, HRV is due to non-linear interactions between number of physiological processes, linearity is a limitation of these time and frequency domain methods, a number of non-linear methods like Poincare' indices, Entropy, generalized and phase synchronization and the methods based on chaos theory have emerged as better tools to analyze HRV. As the non-stationarity of RR interval series (tachogram) limits the use of frequency domain methods based on spectral analysis, new time-frequency techniques have been developed over the time. These include methods like short time Fourier transform, orthonormal basis portioning, wavelet transforms, Wigner ville distribution etc. Using these methods, it is possible to analyze the HRV in time- frequency domain to know how energy of the signals vary with respect to both time as well as frequency. Since, HRV is a result of complex interactions originating from different physiological processes, the complexity of HRV needs to be analyzed.

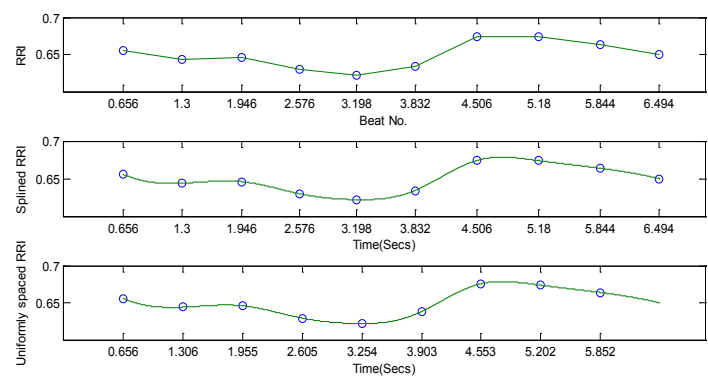

Figure 1. Resampling steps to convert non-uniformly spaced RRi series to a uniformly spaced RRi series.

In this paper, analysis of HRV is performed on age stratified standard Fantasia database from Physionet. This data contains 20 young subjects ( 21 - 34 yrs.) and 20 old subjects (68 - 85 yrs.) strictly - vetted healthy subjects, those experienced 120 mins of uninterrupted supine resting position, whereas, continuous electrocardiogram (ECG), and respiration signals were recorded. In addition, the measurements also included an uncalibrated continuous Arterial Blood Pressure (ABP) waveform. The paper is further distributed in three sections where Section 2 explains the methods used for spontaneous HRV complexity estimation, database used, pre-processing technique employed on the data, Section 3 contains results and discussion followed by conclusion in Section 4.

\section{Methods}

\subsection{Sample}

The Fantasia dataset consists of 20 young and 20 old subjects recorded for HRV studies who watched the 'Fantasia' movie while being recorded. The complexity study was performed on this standard database available at Physionet for each young and old subject. 1000 samples for each RR interval series were used for analysis.

\subsection{Data Pre-processing}

Since, the ECG waveforms is a quasi-periodic waveform, a non-linear algorithm developed using empirical mode decomposition is utilized to detect the R-peaks from the ECG waveform. This method is based on the decomposition of original multicomponent waveform into subsequent Intrinsic Mode Functions (IMFs) of unique instantaneous frequency each. This is a data driven method unlike wavelet transformation which decomposes the input signal based on a pre-defined kernel ${ }^{1,2}$. The ectopic beats were physically removed using the zero degree interpolation technique. The tachogram (RRi series) formed using successive R-R peaks for a typical subject is as shown in Figure 1. The RRi series was normalized according to the following equation:

$R R I_{n}=\frac{R R i-\frac{1}{N} \sum_{i=\mathbf{1}}^{N} R R I(i)}{\frac{\mathbf{1}}{N} \sum_{i=\mathbf{1}}^{N} \operatorname{RRi}(i)}$

where, $\mathrm{N}=$ Number of samples, $i$ corresponds to sample. Beat-to-beat RRi series was utilized for approximate entropy calculation of each subject.

\subsection{Complexity Analysis using ApEn}

For finding sample entropy of a given discrete time series with $\mathrm{N}$ number of sample data points an array of $\mathrm{N}-\mathrm{m}+1$ is formed, here $\mathrm{m}$ is defined as embedding dimension. Every array comprises of $m$ consecutive points and each 
one of the array act as a template for template matching purpose with all datasets. In order to find maximum distance between them and also resulting in formation of conditional vectors having next close value of the position after our selected template set under a pre-decided threshold level referred to as " $r$ "(tolerance also). This process also comprises of template matching with itself also and hence giving rise to bias problem that is $\log 0$ (zero) condition. In the next step, these conditional vectors are arranged as data sets and then conditional probabilities are drawn from each conditional vector. ApEn is defined as:

$$
\operatorname{ApE} n(m, r)=\frac{\sum_{i=1}^{\mathrm{N}-m+1} \ln \left(\frac{C_{i}^{m}(r)}{N-m+1}\right)}{N-m+1}-\frac{\sum_{i=1}^{\mathrm{N}-m} \ln \left(\frac{C_{i}^{m+1}(r)}{N-m}\right)}{N-m}
$$

where, $C_{i}^{m}(r)$ and $C_{i}^{m+1}(r)$ is the conditional probability that the distance between the two vectors lie within the threshold $r$ for window length $\mathrm{m}$ remains within $\mathrm{r}$ for window length $m+1$ respectively.

\subsection{Variation of tolerance threshold ' $r$ '}

Nevertheless, a suggested range of ' $r$ ' has been suggested as (0.1-0.25) times the standard deviation of the HRV time series, in some cases ' $r$ ' within this range may lead to incorrect conclusions in analyzing complexity of a time series. In this paper, ' $r$ ' is varied from 0 to 0.34 in steps of 0.02 and the variation of mean and SD of ApEn is analyzed of HRV for young and old subjects.

\section{Results and Discussion}

Figure 2 shows the variation of mean value of ApEn over 20 young and 20 old subjects as a function of tolerance threshold ' $r$ ' where $r$ is varied from (0.1-0.34) times the standard deviation. It is evident from the results shown in Figure 2 that for $r \geq 0.10$, the complexity of HRV in young subjects is higher than the older ones as depicted by higher ApEn values for young subjects than the old subjects. This is physiologically also correct as the variability in young subjects is more than the older ones. But, as the $\mathrm{r}$ decreases from 0.10 , the trend reverses. Figure 3 shows the variation of SD of ApEn over 20 young and 20 old subjects as a function of tolerance threshold ' $r$ ' where $r$ is varied from (0.1-0.34) times the standard deviation. It is evident from the results shown in the Figure 3, that except for $r<0.02$, the ApEn (SD) is higher in the case of young subjects.

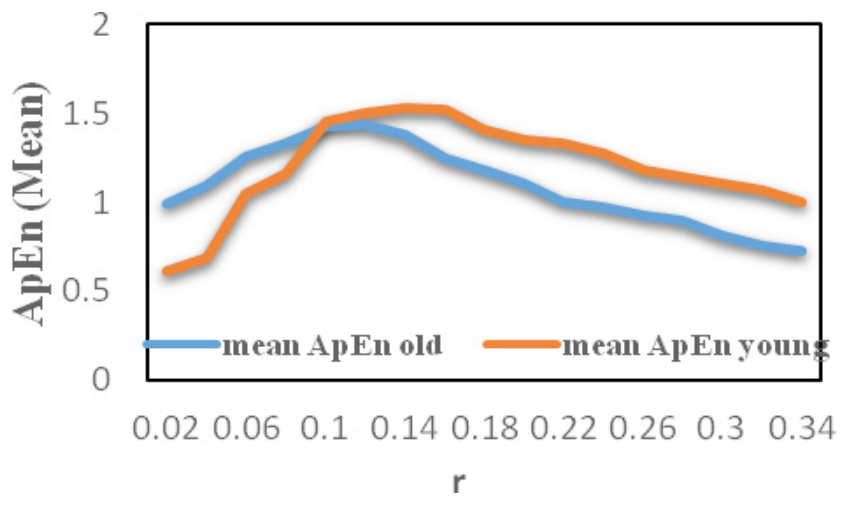

Figure 2. Comparison of mean value of ApEn for young and old subjects over complete spectrum of r.

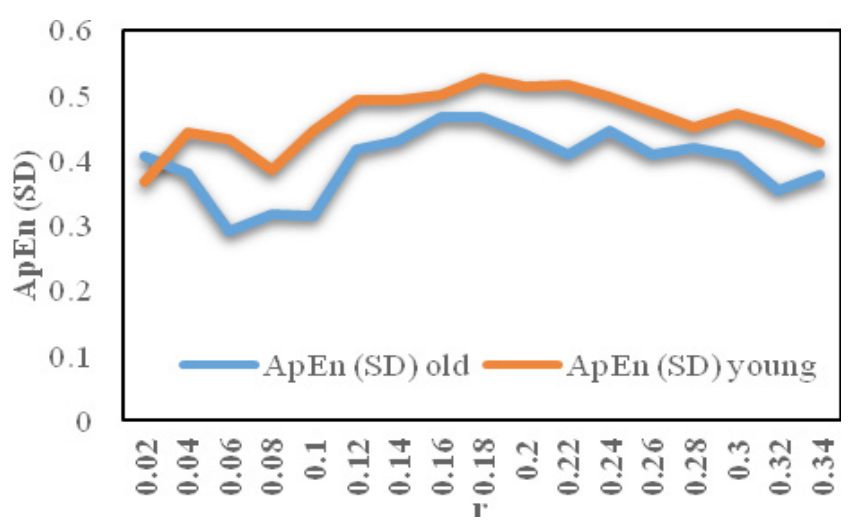

Figure 3. Comparison of SD value of ApEn for young and old subjects over complete spectrum of $\mathrm{r}$.

\section{Conclusion}

Interactions between multiple physiological processes affects the heart rate variability under different physiological conditions. These complex interactions need to be quantified in order to decipher the functioning of autonomic control system. In this paper, the complexity of HRV is analyzed using an information domain indices called ApEn. The analysis was performed on the age stratified data. It is concluded that the complexity of HRV is higher in younger subjects than the older ones. However, the algorithmic parameters like tolerance threshold ' $r$ ' affects these findings. Therefore, the analysis was conducted over a complete spectrum of ' $r$ ' and it is emphasized that the selection of ' $r$ ' is critical for such complexity analysis using ApEn as the trend reverses for certain values of the tolerance threshold ' $r$ ' that leads to misleading conclusion about the true nature of complexity of HRV in younger and older subjects. 


\section{References}

1. Singh A, Saini BS, Singh D. An alternative approach to approximate entropy threshold value (r) selection: application to heart rate variability and systolic blood pressure variability under postural challenge. Medical and Biological Engineering and Computing. 2016; 54(5):723-32.

2. Singh A, Saini BS, Singh D. A new baroreflex sensitivity index based on improved hilbert-huang transform for assessment of baroreflex under supine and standing postures. Biocybernetics and Biomedical Engineering (Elsevier). 2016; 36(2):355-65.

3. Singh A, Saini BS, Singh D. An adaptive technique for Multiscale Approximate $\left(\mathrm{MAE}_{\text {bin }}\right)$ threshold $(\mathrm{r})$ selection: Application to Heart Rate Variability (HRV) and Systolic Blood Pressure Variability (SBPV) under postural stress. Australasian Physical and Engineering Science in Medicine. 2016; 39(2):557-69.

4. Camm AJ, Malik M, Bigger JT, Günter B, Cerutti S, Choen R. Task force of the European society of cardiology and the North American society of pacing and electrophysiology. Heart rate variability-Standards of measurement, physiological interpretation, and clinical use. European Heart Journal. 1996; 17(3):354-81.

5. Singh A, Saini BS, Singh D. Multiscale joint symbolic transfer entropy for quantification of causal interactions between heart rate and blood pressure variability under postural stress. Fluctuation and Noise Letters. 2015; 14(3).

6. Koepchen HP. Physiology of rhythms and control systems: An integrative approach. Rhythms in Physiological Systems. 1991; p. 3-20.

7. Porta A, Di Rienzo M, Wessel N, Kurths J. Addressing the complexity of cardiovascular regulation. Philosophical Transactions of the Royal Society A: Mathematical, Physical and Engineering Sciences. 2009; 367(1892):1215-18.

8. Goldberger AL, Peng C-K, Lipsitz LA. What is physiologic complexity and how does it change with aging and disease? Neurobiology of Aging. 2002; 23(1):23-6.

9. Boettger S, Hoyer D, Falkenhahn K, Kaatz M, Yeragani VK, Bär K-J. Altered diurnal autonomic variation and reduced vagal information flow in acute schizophrenia. Clinical Neurophysiology. 2006; 117(12):2715-22.
10. Goldberger AL. Fractal variability versus pathologic periodicity: complexity loss and stereotypy in disease. Perspectives in Biology and Medicine. 1997 Summer; 40(4):543-61.

11. Di Rienzo, Parati G, Radaelli A, Castiglioni P. Baroreflex contribution to blood pressure and heart rate oscillations: Time scales, time-variant characteristics and nonlinearities. Philosophical Transactions of the Royal Society of London A: Mathematical, Physical and Engineering Sciences. 2009; 367(1892):1301-18.

12. Laude VB, Elghozi JL. Tuning of the sequence technique. IEEE Engineering in Medicine and Biology Magazine. 2009; 28(6):30-4.

13. Deepak KK, Khadka R, Narang R, Jaryal AK, Patel CD, Pandey RM. Baroreflex function appears to initiate compensatory mechanism for cardiac autonomic modulation in patients with angina pectoris. Journal of Physiological Science. 2009; 59:167-76. Sleight P, La Rovere MT, Mortara A, Pinna G, Maestri R, Leuzzi S, Bianchini B, Tavazzi L, Bernardi L. Physiology and pathophysiology of heart rate and blood pressure variability in humans: Is power spectral analysis largely an index of baroreflex gain? Clinical Science. 1995; 88(1):103-10.

14. Di Rienzo M, Parati G, Castiglioni P, Tordi R, Mancia G, Pedotti A. Baroreflex effectiveness index: An additional measure of baroreflex control of heart rate in daily life. American Journal of Physiology-Regulatory, Integrative and Comparative Physiology. 2001; 280(3):R744-51.

15. Deepak KK, Bhargava B, Narang R, Paudel BH, Rai PK, Saxena S. Autonomic studies in hypertensive patient with unusual sexual dysfunction: response to reserpine. Clinical Autonomic Research. 2000; 10(5):313-15.

16. Stratton JR, Levy WC, Caldwell JH, Jacobson A, May J, Matsuoka D, Madden K. Effects of aging on cardiovascular responses to parasympathetic withdrawal. Journal of the American College of Cardiology. 2003; 41(11):2077-83.

17. Kamath MV, Fallen EL. Power spectral analysis of heart rate variability: a noninvasive signature of cardiac autonomic function. Critical Reviews in Biomedical Engineering. 1992; 21(3):245-311. 